Il est de plus en plus clair que la dynamique erratique des marchés financiers est en très grande partie endogène c'est-à-dire qu'elle résulte du fonctionnement interne des marchés et des boucles de rétroaction qui y sont présentes - plutôt que la conséquence de réactions rationnelles à des événements exogènes.

\section{Cette dynamique erratique a}

de nombreux points communs avec celle des systèmes physiques dits « complexes», comme

les aimants désordonnés, où la combinaison hétérogénéitéscompétition conduit de

manière générique à de longues périodes de stases interrompues par des crises violentes, et à une hypersensibilité à de faibles changements des paramètres extérieurs. Ces systèmes

physiques pourraient s'imposer comme des métaphores utiles de la complexité des systèmes économiques.

Cet article est une version élaborée d'un article paru en langue anglaise dans Physics World 22, n4 (2009), pp. 28-32.

\title{
La (regrettable) complexité des systèmes économiques
}

Jean-Philippe Bouchaud (jean-philippe.bouchaud@cea.fr)

Capital Fund Management, 6 bd Haussmann, 75009 Paris et École polytechnique, 91128 Palaiseau Cedex

La crise actuelle met la pensée économique classique sous pression extrême ; j'espère d'ailleurs qu'elle ne s'en remettra pas. La théorie classique veut qu'un marché complètement dérégulé atteigne un équilibre " efficient ", qui permet une allocation optimale des ressources, et dont les prix reflètent parfaitement, de manière non biaisée, toute l'information disponible. De tels marchés devraient être stables : les crises ne peuvent apparaître qu'à l'occasion de chocs exogènes, mais jamais à cause de leur dynamique interne, qui serait faite de petites fluctuations - supposées gaussiennes rapidement éteintes grâce à l'action d'agents rationnels prompts à " arbitrer ", c'est-à-dire tirer profit de ces erreurs d'appréciations en les faisant disparaître.

On mesure depuis quelques mois l'ineptie d'un tel cadre théorique qui, pourtant, depuis sa formalisation mathématique des années 50 et 60 , a revendiqué haut et fort le statut de théorie scientifique. Sa supposée victoire théorique (contre Keynes en particulier, dont la profondeur de pensée reste incroyablement actuelle) a été telle que plusieurs générations de dirigeants et de conseillers en ont fait leur vade-mecum, justifiant une politique de dérégulation des marchés de plus en plus agressive depuis une trentaine d'années, et qui est partiellement responsable de la crise. Toute contrainte est en effet censée éloigner les marchés de leur équilibre parfaitement efficient.

Alan Greenspan lui-même avouait son désarroi en novembre dernier: "Those of us who have looked to the self-interest of lending institutions to protect shareholders' equity, myself included, are in a state of shocked disbelief", avant d'ajouter: "Yes, I've found a flaw in the theory. I don't know how significant or permanent it is. But I've been very distressed by that fact."
Il parlait de la théorie des marchés efficients, qui lui semblait tout d'un coup infondée.

La théorie des agents rationnels a pourtant étendu son emprise sur d'autres domaines - aussi effarant que cela puisse paraître, il existe des théories rationnelles du mariage, du chômage, de l'obésité, de la toxicomanie, etc. Ce n'est pas le lieu de développer cette polémique ; mais, du point de vue de ses conséquences politiques et sociales, l'idée de l'homme infiniment rationnel, infaillible et donc irrémédiablement responsable de ses choix, me semble à titre personnel regrettable et terriblement dangereuse.

Mais cette idée est surtout non fondée scientifiquement, pour un certain nombre de raisons que je voudrais détailler. Lors de la première conférence de Santa Fe regroupant physiciens et économistes, Phil Anderson a émis son fameux "Do you guys really believe that ?", qui résume assez bien la réaction des physiciens à ces hypothèses qui paraissent très peu plausibles et choquent l'intuition. En réalité, un certain nombre d'économistes sont eux-mêmes convaincus que ces hypothèses sont inadéquates. Pourtant, le mythe perdure et les manuels ont du mal à évoluer. Un argument souvent avancé est qu'il vaut mieux garder une hypothèse fausse qui permet le développement d'une théorie mathématique rigoureuse, plutôt que de s'aventurer dans la modélisation de l'infinie manière dont les agents pourraient ne pas être rationnels. Il me semble pourtant primordial que la modélisation en économie et en sciences sociales cherche à décrire l'homme tel qu'il est et non tel qu'il devrait être selon la théorie - émotif et capable d'erreurs plutôt que calculateur et infaillible. 


\section{6}

Il me semble primordial que la modélisation en économie et en sciences sociales cherche à décrire l'homme tel qu'il est et non tel qu'il devrait être selon la théorie - émotif et capable d'erreurs plutôt que calculateur et infaillible.

\section{Dynamique exogène ou endogène?}

L'un des points les plus importants à comprendre, remis sur le devant de la scène en cette période de crise, est celui de la nature endogène ou exogène des fluctuations des marchés. Dans un marché efficient au sens informationnel, les prix reflètent la valeur intrinsèque des actifs, qui n'évoluent que sous l'effet de nouvelles exogènes. Un krach boursier ne peut donc se produire que si les conditions extérieures au système économique lui-même changent brutalement. Par exemple, un tremblement de terre à Tokyo qui détruit matériellement les infrastructures d'une société. De manière moins spectaculaire, le prix d'une société cotée ne devrait changer significativement que lorsque tombe une nouvelle concernant les perspectives futures de son activité.

Plusieurs travaux ont cherché à tester cette idée quantitativement, avec des résultats négatifs. Par exemple, Cutler, Poterba et Summers en 1989, dans What moves stock prices?, concluent qu'il est difficile d'attribuer la volatilité des marchés à des nouvelles macroéconomiques ou politiques. Robert Shiller a démontré l'innocuité des nouvelles qui entourent le " grand krach » de 1987. Plus récemment, nous avons mené une étude exhaustive des changements de prix des actions, échantillonnés à la minute et syn-

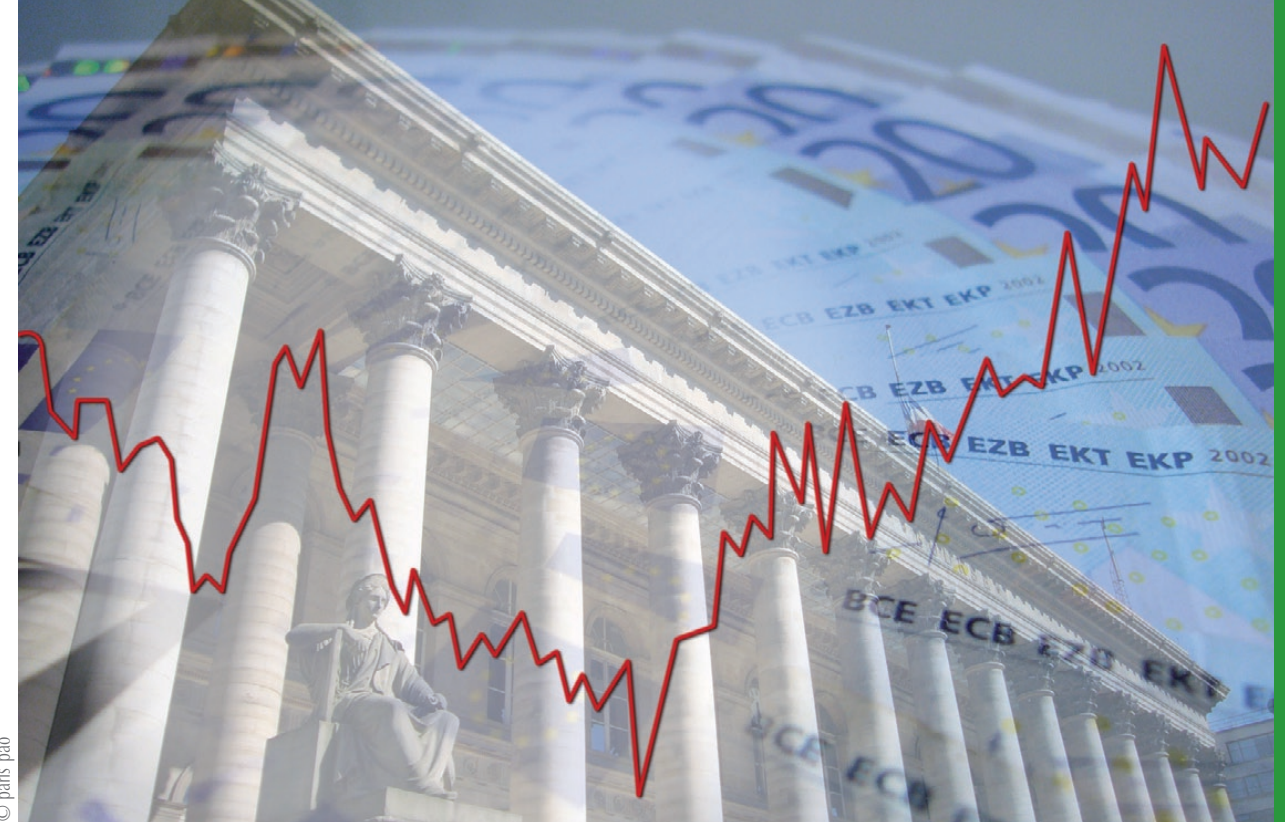

chronisés avec le flux de dépêches. Nous avons trouvé que seulement 5\% des sauts importants peuvent être attribués à une nouvelle concernant la société en question ; la majorité des sauts semblent être induits par la dynamique intrinsèque des marchés eux-mêmes. D'ailleurs, les deux types d'événements conduisent à des "répliques " (au sens des tremblements de terre) de signatures statistiques très différentes, ce qui montre bien qu'ils sont de nature distinctes.

De manière plus globale, la statistique des variations de prix montre des spécificités qui ne sont pas naturelles dans le contexte de l'équilibre : distribution de Pareto (en loi de puissance, $\mathrm{x}^{-\alpha}$ ) et donc fortement non gaussienne des variations de prix, intermittence multi-échelle de la volatilité (fig. 1), etc. La nature non gaussienne des fluctuations remet très sérieusement en cause la validité d'un standard de l'industrie financière : l'équation de Black-Scholes des options ou, de manière plus crue, le mythe du risque nul, qui sous-tend l'approche (trop) classique des mathématiques financières de valorisation par réplication parfaite (cf. encadré 1, p. 25), opération uniquement possible sous des hypothèses très fortes de fluctuations gaussiennes qui permettent en effet de se débarrasser du risque. Cette approche, encore dominante dans les manuels et les esprits, conduit à une mauvaise intuition et une déresponsabilisation vis-à-vis du risque. L'ironie est que l'utilisation de l'équation de Black-Scholes, qui néglige la probabilité des grands mouvements, a eu comme conséquence d'amplifier considérablement le krach de 1987 par un effet de rétroaction positive des ordres de vente sur les prix !

\section{Impact et boucles de rétroaction}

Un effet crucial ici, négligé (à vrai dire escamoté) par la théorie économique classique, est l'impact des transactions sur les prix. Dans la vision classique, la valeur fondamentale est fixée en dehors des marchés eux-mêmes, qui ne font que la révéler en agrégeant l'information des opérateurs. En aucun cas les transactions «non informées » ne devraient affecter les prix, sinon on observerait un découplage entre valeur fondamentale et prix du marché.

L'accès aux données de ultra-haute fréquence (en dessous de la seconde), qui permet de suivre l'évolution du marché ordre par ordre, montre que chaque transaction, informée ou non, impacte les prix faiblement, certes, mais de manière systématique et mesurable (l'ordre de grandeur est de quelques $\%$ de $\%$ pour $1 \%$ du volume quotidien). L'existence de cet impact complète et renforce l'argument selon lequel les fluctuations de prix sont principalement endogènes. L'impact des transactions peut faire naitre des boucles de rétroactions. Par exemple, l'utilisation du modèle de Black-Scholes conduit mécaniquement, dans certains cas, à une avalanche d'ordres de vente lorsque le cours baisse - précisément ce qui s'est passé en 1987. Ces boucles de rétroaction déstabilisantes sont monnaie courante, si l'on ose dire, et les plus embarrassantes sont celles qui sont dues à la théorisation des marchés ; la crise actuelle est, à nouveau, une illustration d'école de la nocivité potentielle des dogmes et de la modélisation. La sousestimation du risque de défaut collectif d'un grand nombre d'entreprises dans les modèles de dérivés de crédit (les fameux CDOs) ont contribué à engendrer la crise, et donc le défaut collectif lui-même !

\〉 

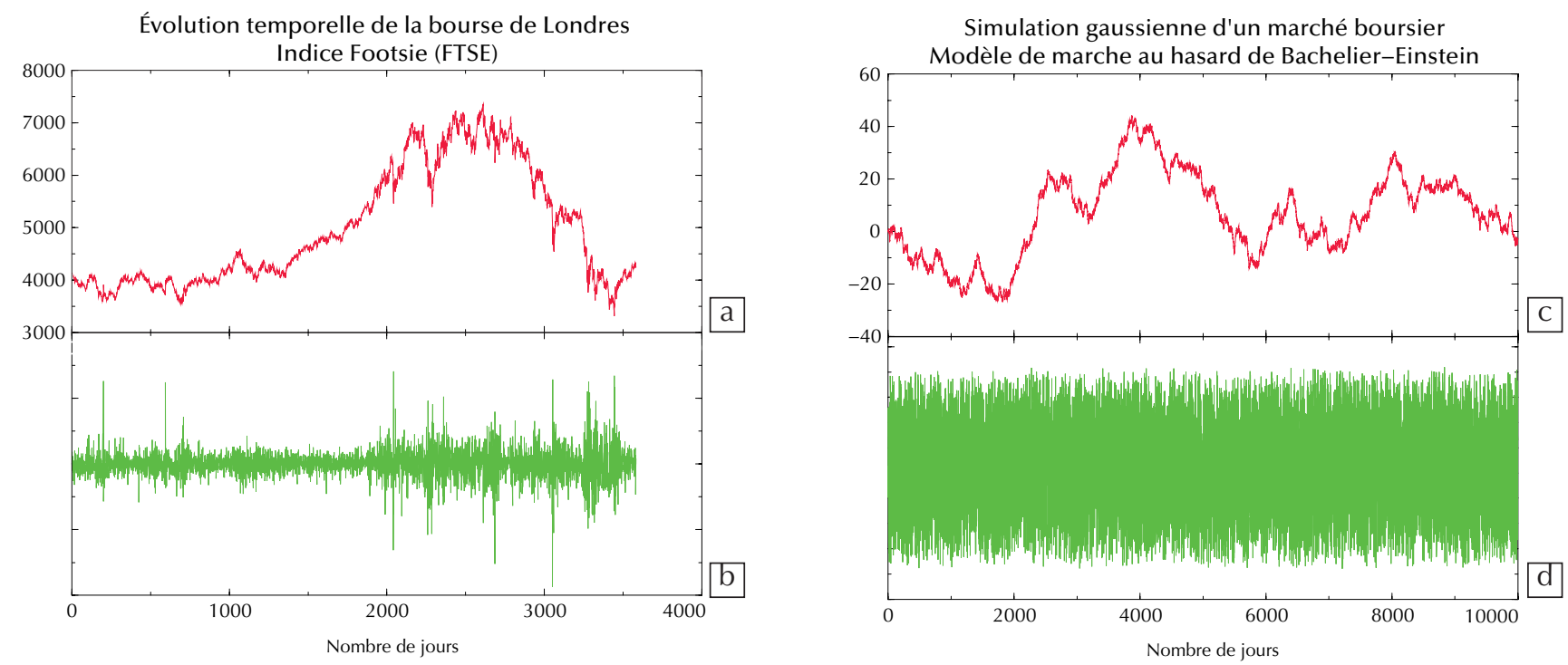

1. Marchés financiers : le Footsie et le mouvement brownien de Bachelier. On montre deux façons de représenter l'évolution temporelle des prix : le prix en fonction du temps (figs 1-a et 1-c), et sa « dérivée ", c'est-à-dire les variations quotidiennes du prix, toujours en fonction du temps (figs 1-b et 1-d). Les deux cas considérés sont l'indice Footsie (FTSE) de la bourse de Londres (figs 1-a et 1-b), et les mouvements de prix synthétiques en supposant le modèle de mouvement brownien de Bachelier et Black \& Scholes (figs 1-c et 1-d). Les différences entre le modèle et la réalité apparaissent à l'œil nu. Les « pics » observés sur la figure 1-b correspondent aux grands mouvements de prix (à la hausse ou à la baisse), qui sont absents dans le modèle gaussien. La nature intermittente et multi-échelle des fluctuations est aussi apparente : les grands mouvements se produisent en grappe, et ces épisodes peuvent aussi bien durer une minute que dix ans (comme après la crise de 1929) ! La nature intermittente de la dynamique des prix suggère des analogies possibles avec d’autres systèmes complexes étudiés par les physiciens : la turbulence, le bruit Barkhausen (fig. 2), le mouvement des fissures dans les matériaux hétérogènes ou d'une ligne de contact sur un substrat rugueux.

\〉

\section{Analogies physiques}

Revenons au caractère « anormal » de la statistique des fluctuations de prix : distribution en loi de puissance des variations de prix, intermittence multi-échelle de la volatilité, etc. Une observation très importante est l'universalité de ces résultats : il semble que quel que soit l'actif traité actions, devises, matières premières, ou même la volatilité implicite sur les marchés d'options (voir encadré 1), à Londres ou à Tokyo, il y a 200 ans sur les marchés de céréales ou maintenant - on observe à peu près les mêmes caractéristiques. La phénoménologie empirique, en particulier les lois de puissance et la dynamique intermittente, frappe l'imagination du physicien statisticien car de nombreux systèmes complexes partagent cette phénoménologie, et le point crucial est que cette dynamique intermittente est purement endogène. Citons par exemple les écoulements turbulents, la progression d'un front de fissure dans un matériau désordonné, le retournement de l'aimantation dans un ferromagnétique désordonné (bruit dit de Barkhausen, figure 2). Dans tous ces exemples, le forçage extérieur, la dynamique imposée, est lente et régulière, mais la dynamique réalisée est, elle, irrégulière, intermittente, faite de périodes calmes et statiques interrompues par des bouffées instables, des avalanches d'activité. Par exemple, le front de fissure se coince tem- porairement avant d'avancer par à coups. Même si comparaison n'est pas raison, ces analogies sont frappantes.

Je voudrais discuter la façon dont des éléments, à mon avis essentiels, laissés de côté dans la modélisation classique de l'agent rationnel, pourraient assez naturellement conduire à une dynamique intermittente et " chaotique », endogène aux marchés.

Un premier ingrédient crucial est l'imitation dans la prise de décision. La transcription du "modèle d'Ising en champ aléatoire " (RFIM, voir encadré 2, p. 26) permet de décrire des situations où il y a conflit entre opinions individuelles, information publique et pression sociale. Imaginons un ensemble d'agents qui ont des opinions a priori divergentes, par exemple optimistes (acheteurs) et pessimistes (vendeurs), avec toutes les gradations intermédiaires. Ces agents aux opinions hétérogènes sont par ailleurs influencés par des facteurs globaux qui évoluent lentement, par exemple les taux d'intérêt, l'inflation, les prévisions de dividendes futurs, etc. On suppose qu'il n'y a pas de " chocs", de discontinuités dans l'évolution de ces facteurs exogènes. Mais on postule que nos agents sont influencés par l'opinion de la majorité, ce qui semble un trait psychologique assez évident, amplifié par une pratique généralisée de jugement par comparaison, surtout en finance : on est toujours pardonné d'avoir eu tort avec la masse, et il est parfois difficile de justifier que l'on puisse avoir raison tout seul. Bref, dans ce modèle, on adopte l'opinion de la majorité si sa propre conviction a priori est plus faible que sa propension à l'imitation grégaire.

Si tous les agents prenaient leur décision dans la solitude de l'isoloir, sans prêter attention à ce que pensent les autres (donc une propension à l'imitation nulle), l'opinion agrégée suivrait fidèlement les influences externes et, par hypothèse, évoluerait continûment. Mais de manière surprenante, si cette propension à l'imitation dépasse un certain seuil critique, l'évolution de l'opinion moyenne peut sauter brutalement, de façon discontinue, de l'optimisme vers le pessimisme, alors que les facteurs extérieurs se dégradent progressivement et continûment. De plus, une hystérèse apparaît. De la même façon que la vapeur saturée refuse de se transformer en liquide, l'état optimiste est maintenu de manière auto-référentielle, bien au-delà du point où les facteurs globaux auraient dû conduire au pessimisme. Lors de l'amélioration, l'optimisme revient lui aussi avec retard, personne ne voulant y croire parce que personne n'y croit.

Bien sûr, ce modèle est extrêmement simplifié, mais on ne peut pas ne pas voir de fortes ressemblances avec toutes les bulles de l'histoire économique et financière. La prise de conscience progressive des montagnes de dettes accumulées par les banques ces 
dernières années aurait dû conduire à une correction progressive des marchés, un " atterrissage en douceur ", comme prévu par la théorie des marchés efficients. Au contraire, l'euphorie collective a perduré, faisant fi de tous les signes inquiétants avant de devenir insoutenable. La moindre étincelle, un événement anecdotique peut déclencher une crise d'une ampleur incommensurable à sa cause, comme en 1987 ou en 2008.

Le modèle RFIM illustre l'impossibilité de représenter l'évolution de l'opinion moyenne, ou de la demande agrégée d'une population hétérogène, par celle d'un agent représentatif. Cette hypothèse est pourtant souvent invoquée pour passer de la modélisation des agents individuels au comportement "macroscopique ", comme on dirait en physique, où le passage micro-macro est un thème récurrent et un problème en général difficile, surtout dans les matériaux hétérogènes !

\section{Utilité et complexité}

Je voudrais enfin revenir sur la modélisation classique des comportements individuels, en termes d'agents dits " rationnels». Ce point de départ est décrit récemment par Ivar Ekeland comme le " cheval de bataille » de l'économie moderne. On suppose que les décisions prises sont le résultat d'un calcul qui permet à l'agent rationnel de maximiser sa "fonction d'utilité ", censée mesurer sa satisfaction. Il ne s'agit pas pour lui de se contenter de solutions acceptables, mais bien de trouver le maximum absolu de cette fonction.

On peut opposer à cette idée un grand nombre de critiques. Une des plus profondes me paraît concerner le processus de maximisation lui-même. Il semble en effet que les défenseurs de cette théorie aient en tête des fonctions d'utilité que l'on peut qualifier de "simples ", par exemple un paraboloïde à maximum unique, et faciles à déterminer - faciles dans un sens algorithmique. Dans cet exemple, une montée suivant le gradient local conduit rapidement au maximum, le temps de calcul croît lentement avec le nombre de variables $\mathrm{N}$ et il est plausible que l'esprit humain soit capable d'accomplir une telle tâche. Mais le problème est que ce type de fonction représente l'exception plutôt que la règle, et que de nombreux problèmes d'optimisation sous contrainte ne sont pas de ce type. La physique des verres de spin nous a en effet fait comprendre que, de manière générique, l'énergie (analogue

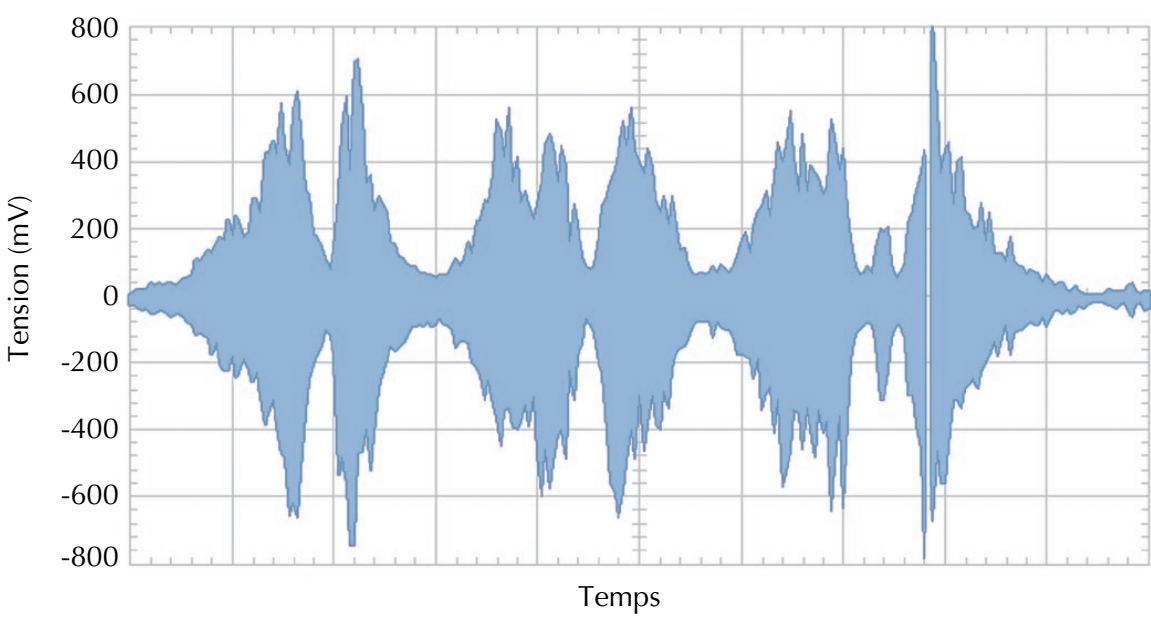

2. Trace temporelle du « bruit Barkhausen " lors de l'aimantation d'un matériau magnétique désordonné soumis à un champ magnétique extérieur. Le champ tend à faire bouger les parois entre les domaines + et les domaines - pour faire disparaître les domaines dont l'aimantation lui est opposée. Le bruit Barkhausen est causé par le mouvement intermittent de ces parois lorsqu'elles se dépiègent des impuretés, puis se repiègent à un temps ultérieur. Cette dynamique est une succession d'avalanches multi-échelles, qui correspondent à des volumes de toutes tailles balayés par les parois lors de leur décrochement (voir J.P. Sethna et al., Nature 410 (2001) 242).

\section{Options - La réplication parfaite de Black et Scholes}

Une option est une sorte d'assurance contre les fluctuations des marchés financiers. Par exemple, on peut vouloir assurer son portefeuille boursier contre un effondrement des cours, et ainsi acheter une " option » qui fournit la garantie d'un prix de rachat minimum de ses actions. De nombreux produits de ce type sont en fait, de façon parfois cachée, déjà disponibles pour le grand public, et portent le nom d'investissements à capital garanti. Dans ce cas, la valeur minimale garantie est le capital initial.

Quel est le montant raisonnable de la prime d'assurance à laquelle doit consentir l'acheteur de l'option ? Le vendeur d'option peut-il suivre une stratégie optimale d'achat et de vente du " sous-jacent » (c'est-à-dire l'objet sur lequel porte l'option) de façon à minimiser son risque? Il est clair que la réponse à ces deux questions nécessite une description de la dynamique du sous-jacent, un modèle probabiliste de son évolution future. Black et Scholes, en 1973, proposent une solution complète du problème en postulant que le logarithme du prix effectue un mouvement brownien.

La formule qu'ils obtiennent exprime la valeur de l'option comme une fonction de la volatilité du sous-jacent, c'est-à-dire sa propension à fluctuer, son coefficient de diffusion comme on dirait en physique. Les marchés d'options sont ainsi des marchés où s'échangent des anticipations de volatilité future, appelée " volatilité implicite » par les traders options. Mais le résultat le plus frappant de Black et Scholes est que la stratégie optimale est parfaite, dans le sens où I'assureur ne prend aucun risque! Autrement dit, la stratégie de couverture permet une réplication parfaite de l'option, puisque les fluctuations de valeur de l'option sont exactement compensées par celles de la stratégie de couverture. Mais ce résultat est intimement lié à la nature supposée gaussienne des fluctuations et à la limite (irréaliste) du temps continu, où la fréquence des transactions tend vers l'infini. En présence d'effets non gaussiens (queues de distributions, volatilité stochastique, voir fig. 1), la possibilité de trouver une stratégie parfaite disparaît, et il subsiste un risque résiduel, qui représente en pratique une fraction très importante de la valeur de l'option. 
$>>$

de la fonction d'utilité) possède un nombre très grand, exponentiel en $\mathrm{N}$, d'extrema locaux, et qu'un très grand nombre d'entre eux sont quasi dégénérés.

On est face à un mur exponentiel de complexité. Le maximum absolu est non seulement très difficile à trouver - le temps nécessaire aux meilleurs algorithmes est lui aussi exponentiel en $\mathrm{N}-$, mais en plus il est à peine meilleur qu'une nuée d'autres maxima locaux. Ce maximum absolu est de surcroît très instable par rapport à de petits changements des paramètres qui déterminent la fonction d'utilité : la position relative des différents maxima locaux s'inverse très facilement. On est dans une situation similaire à celle du chaos des systèmes dynamiques, où une incertitude change la trajectoire et où la maîtrise de celle-ci demande une précision exponentielle. Le maximum absolu n'a donc pas de vertu particulière, et le maximum " acceptable " dépend énormément de l'heuristique, de l'algorithme utilisé pour le trouver.

Dans ce contexte, la théorie de l'agent maximisant me semble en difficulté : comment imaginer que le cerveau humain puisse réaliser rapidement des opérations que les plus puissants ordinateurs, avec les meilleurs algorithmes, sont incapables de faire ? Comment imaginer que tout se passe " comme si " les agents trouvaient leur maximum absolu, alors que celui-ci est intrinsèquement instable ? Ou bien la théorie de l'agent rationnel n'est-elle valable que pour des situations suffisamment simples? Dans le cas générique, la rationalité est donc de facto limitée, la décision finale (qui peut différer notablement dans ses conséquences) dépend de l'algorithme, de l'histoire, du choix précis des paramètres, etc. Même si le problème est intrinsèquement déterministe, il apparaît un aléa qui provient de ce choix incertain, qui fait boule-de-neige - puisque les stratégies individuelles ne peuvent plus supposer la rationalité auto-réflexive et infinie des autres agents.

Plus généralement, les systèmes économiques dans leur ensemble partagent un grand nombre des ingrédients qui sont à la base de la complexité exponentielle des systèmes de type "verres de spin " en physique : de nombreuses entités hétérogènes en compétition forte. La dynamique de ces systèmes est particulièrement intéressante et a fait l'objet d'études nombreuses ces dernières années. À cause du très grand nombre de minima locaux de l'énergie qui piègent temporairement le système, son évolution est très lente, avec de longues périodes de stases séparées par de rapides changements de configuration dominante. Le système n'atteint l'équilibre qu'au bout d'un temps extrêmement long, parfois bien plus long que les temps géologiques.

Si la métaphore "verres de spin " s'appliquait aux systèmes économiques, un changement profond de paradigme s'imposerait alors :

- la notion d'équilibre, chère aux économistes, perdrait complètement son intérêt puisque le temps d'équilibre serait beaucoup trop long (les verres n'atteignent pas l'équilibre, même après des temps géologiques !) ;

- la dynamique hors équilibre, thème à ma connaissance assez peu abordé par l'économie classique, deviendrait alors le sujet pertinent ;

\section{Magnétisme désordonné : champs aléatoires et verres de spin}

Encadré 2

Deux modèles phares ont été introduits pour décrire le magnétisme d'alliages désordonnés : d'une part, le modèle d'Ising en champ aléatoire (RFIM en anglais), dans lequel chaque spin interagit de façon ferromagnétique avec ses voisins mais est soumis à un champ de signe aléatoire qui tend à rompre l'ordre ferromagnétique ; d'autre part, le modèle d'Edwards-Anderson (EA) des verres de spin, dans lequel l'interaction elle-même est de signe aléatoire, conduisant à un ordre amorphe. L'énergie d'une configuration des $N$ spins $S_{i} s^{\prime}$ écrit : $H=-\Sigma_{\mathrm{i}} h_{\mathrm{i}} S_{\mathrm{i}}-\Sigma_{\mathrm{ij}} j_{\mathrm{ij}} S_{\mathrm{i}} S_{\mathrm{j}}$, avec $J_{\mathrm{ij}}=J>0$ et $h_{\mathrm{i}}$ aléatoire pour le RFIM, et avec $J_{\mathrm{ij}}$ aléatoire et $h_{\mathrm{i}}=0$ pour le modèle EA.

Ces modèles ont été l'objet d'études analytiques et numériques intensives au cours des trente dernières années, et sont devenus des paradigmes théoriques pour la compréhension des systèmes complexes, bien au-delà des problèmes magnétiques qui leur ont donné naissance. Ils encodent de manière minimale les effets de compétition entre interaction et hétérogénéité. Un des aspects les plus importants, qui confère à ces modèles une richesse extraordinaire (duale d'une difficulté théorique redoutable) est l'existence d'un nombre exponentiel de configurations métastables, c'est-à-dire de minima locaux de l'énergie $H$.
- les systèmes économiques complexes seraient alors fragiles et sensibles à de petites perturbations, et évolueraient de manière intermittente, en une succession d'époques quasi stables séparées par des "crises " imprévisibles, et ce même si les influences extérieures évoluent lentement et continûment. De " grosses » nouvelles ne sont pas nécessaires pour faire tanguer de tels marchés ; leur instabilité est intrinsèque et vient de la compétition même entre agents hétérogènes.

\section{Conclusion}

Les modèles que je viens d'évoquer sont plus inspirants que convaincants. Ils restent, à ce stade, assez vagues et doivent être approfondis - en particulier en utilisant des simulations numériques de modèles d'agents pour construire des marchés artificiels réalistes. Mais ils racontent des histoires qui me semblent suffisamment intéressantes pour qu'on les prenne au sérieux. L'idée suivant laquelle le mélange compétition/hétérogénéités permet d'expliquer en grande partie les crises endogènes des marchés et des systèmes économiques, me paraît assez plausible pour mériter qu'on s'y attarde.

En attendant, il me semble que l'état d'esprit dans lequel la science économique s'est développée et a irrigué l'ingénierie financière doit évoluer. Les innovations financières doivent être analysées de manière critique, testées et réglementées par une agence indépendante et dotée d'une autorité, comme le sont les innovations dans d'autres industries à risque (nucléaire, chimique, pharmaceutique, aéronautique, etc.) : pourquoi l'industrie financière y échapperait-elle, à part pour des arguments de mauvaise foi dont la justification ultime est la recherche du profit maximum à court terme ? Mon avis est que la crise actuelle, qui a de graves répercussions sur le quotidien des gens, justifie le parallèle avec ces activités humaines dangereuses. Je pense que les préjugés hérités d'une éducation trop formelle, dogmatique et éloignée du réel font partie du problème. Une représentation mentale plus pragmatique, où l'intuition et les mécanismes remplacent l'axiomatisation et les modèles formels, et l'enseignement d'une science économique redevenue avant tout, et farouchement empirique, me semblent être des prérequis pour une stabilité du monde financier sur le long terme. 